https://doi.org/10.18778/2196-8403.2020.09

SANDRA MÜLLER

\title{
Der „Anschluss“ Österreichs 1938 in aktuellen österreichischen Schulgeschichtsbüchern
}

Der Aufsatz beschäftigt sich mit der Darstellung des ,Anschlusses ‘ Österreich 1938 am Beispiel von Schulbüchern. Dazu werden aktuell in Österreich für den Schulgebrauch zugelassene Schulbücher aspektorientiert mit Blick auf Kapitelstrukturen, Konzeptorientierung, Deutungsmuster und Textquellen- und Bildverwendung analysiert. Die Analyse hat einen ambivalenten Umgang mit dem Anschluss ergeben.

Schlüsselwörter: Schulbücher, Geschichtsunterricht, Schulbuchforschung, Geschichtspolitik

The representation of the annexation of Austria 1938 in recent Austrian textbooks for history teaching

The article examines how the 'Anschluss' of Austria in 1938 is depicted in current history textbooks. The textbooks are analysed by looking at chapter structure, underlaying concepts, and the use of pictures and sources. The analysis showed an ambivalent portrayal of the events of 1938 .

Keywords: textbooks, history teaching, textbook research, politics of memory

'Anschluss' Austrii w 1938 r. w aktualnych austriackich podręcznikach do historii Artykuł prezentuje, w jaki sposób podręczniki szkolne przedstawiają 'Anschluss' Austrii z 1938 roku. Analizie poddane zostały książki aktualnie dopuszczone do kształcenia. Autorka stawia pytania o poszczególne cechy podręczników, takie jak struktura rozdziałów, koncepcja całości, wzory interpretacji i źródła tekstowe a także funkcja ilustracji. Analiza wykazała, że podręcznikowy przekaz o 'Anschlussie' w szkołach austriackich nie jest jednoznaczny.

Słowa klucze: podręczniki, lekcja historii, badanie podręczników, polityka historyczna 


\section{Einleitung}

„Alle Sachverständigen sind sich darüber einig, daß das Schulbuch das wichtigste Medium des Geschichtsunterrichts ist" (RÜSEN 1994:156). An dieser Feststellung des Geschichtsdidaktikers Jörn Rüsen aus dem Jahr 1994 hat sich bis heute wenig geändert, obwohl die Bedeutung von Schulgeschichtsbüchern gelegentlich angezweifelt wird (vgl. OLESCHKO / MORAITIS 2012:18). Das Schulbuch gilt trotz steigender Zahlen von alternativen Angeboten im Bereich der Open Educational Resources (OER) nach wie vor als „Leitmedium des Geschichtsunterrichts“" (RÜSEN 2008:162). Geschichtsunterricht wiederum - und mit ihm die verwendeten Schulbücher - prägt das Geschichtsbewusstsein junger Menschen entscheidend mit (vgl. BORRIES 1995a:293-298). Der schulische Geschichtsunterricht legt ein Fundament für das Geschichtsbewusstsein von Erwachsenen und damit dafür, wie sie durch „Sinnbildung über Zeiterfahrung“ (RÜSEN 1983:50) die Gegenwart analysieren, die Vergangenheit deuten und Erwartungen an die Zukunft herantragen (vgl. PANDEL 2017:135). Gleichzeitig strukturieren Schulgeschichtsbücher als Leitmedien Unterricht und legen nahe, welche Text- und Bildquellen herangezogen werden, auf welche Unterthemen der Fokus gelegt wird. Sie ermöglichen dabei unterschiedlich intensive Nutzungsweisen, vom bloßen Informieren über Sachverhalte bis hin zur tiefgreifenden Erarbeitung und Überprüfung von Hypothesen (vgl. BECHER 2017:45). Das Schulgeschichtsbuch ist immer auch ein geschichtspolitisches Medium, wenn man den Begriff der Geschichtspolitik nach Edgar Wolfrum zugrunde legt, der Geschichtspolitik als „Handlungs- und Politikfeld [versteht], auf dem verschiedene Akteure Geschichte mit ihren spezifischen Interessen befrachten und politisch zu nutzen suchen“(WOLFRUM 1999:25; vgl. BECKER 2014:51-69), indem sie Deutungszusammenhänge herstellen. Schulbücher stellen solche $\mathrm{Zu}-$ sammenhänge her durch die Abbildung und Vermittlung dessen, was von einer Gesellschaft bzw. einer gesellschaftlichen Gruppe als relevante Ereignisse in der Vergangenheit verstanden wird, wie diese Ereignisse mit Deutungen aufgeladen werden und wie Schülerinnen und Schüler mit diesen umgehen sollen. Die Akteure, die sich an der geschichtspolitischen Aufladung von Schulbüchern beteiligen, sind dabei Autorinnen und Autoren, Verlegerinnen und Verleger sowie Politikerinnen und Politiker (vgl. SCHÖNEMANN / THÜNEMANN 2010:9f.). Schulgeschichtsbücher sind in den Inhalten zwar rückwärtsgewandt, da sie Vergangenheit darstellen und vermitteln sollen, sagen aber mindestens ebenso viel über die Gegenwart aus, in der sie entstehen, und das Geschichtsverständnis der beteiligten geschichtspolitischen Akteure, die sie zu verantworten haben 
(vgl. HAMMERSTEIN 2017:30; JACOBMEYER 1998:31). Bis zu ihrer Zulassung durchlaufen sie verschiedene Überprüfungen hinsichtlich inhaltlicher und didaktischer Qualität, aber auch hinsichtlich politischer Fragen. Durch ministeriale Vorgaben und Kontrollen sind Schulbücher letztlich den Spielregeln der Politik unterworfen, gleichsam ist der Geschichtsunterricht „eine der wichtigsten Instanzen der politischen Bildung" (RÜSEN 1994:157). Schulgeschichtsbücher sind somit nicht nur Medien des Geschichtsbewusstseins und der Geschichtskultur, sondern auch der Geschichtspolitik.

Der Wert von Schulbüchern für eine Bilanz zur Erinnerungskultur 1938-2018 in Österreich liegt nicht darin abzulesen, wie Schülerinnen und Schüler Erinnerungskultur deuten oder sie ,als passive Quelle des Geschichtsunterrichtes“ (MITTNIK / KÜHBERGER 2015:10; vgl. MITTNIK 2017) verstehen. Dazu wären empirische Studien zur Rezeption von Schulbüchern nötig, wie sie an anderer Stelle bereits angestellt wurden (vgl. MITTNIK / KÜHBERGER 2015:10-13; OLESCHKO / MORAITIS 2012:14-16; BORRIES 1995b:45-60; BORRIES 2005). Vielmehr liegt der Wert von Schulgeschichtsbüchern für eine solche Bilanz darin, erforschen zu können, wie das Schlüsseljahr 1938 im ,Idealfall', im Sinne des staatlich gewollten Geschichtsbildes und der eigenen Nationalgeschichte vermittelt und dargestellt werden soll (vgl. MARKOVA 2015b:119f.). Oder, um es mit Wolfgang Jacobmeyer auszudrücken: es soll untersucht werden, inwiefern die Schulgeschichtsbücher in dieser Hinsicht als eine „Autobiografie der Nation“ (JACOBMEYER 1998) fungieren. Dieser Beitrag bezieht sich dabei auf eine Stichprobe aus aktuell in Österreich zugelassenen Schulbüchern für die allgemeinbildenden höheren Schulen (AHS) und ist somit nicht im Sinne einer diachronen Bilanz, sondern einer aktuellen Bestandsaufnahme zu verstehen. In den Blick genommen wurden Schulbücher für die 4. Klasse AHS Unterstufe und die 7. Klasse AHS Oberstufe, da das Thema Nationalsozialismus für diese Schuljahre durch den Lehrplan vorgeschrieben ist. Nach der ministeriellen Zulassung der Schulbücher für den Unterrichtsgebrauch wählen die Schulen aus der Liste der zugelassenen Schulbücher aus und bestellen diese zentral.

Neben diesen Rahmenbedingungen in der Erstellung und Beschaffung von Schulbüchern ist ein Blick in die Vorgaben des Lehrplans zur Einordnung der Schulbuchanalyse unabdingbar.

Für den Umgang mit dem Nationalsozialismus in Österreich waren besonders die Lehrplanrevisionen aus den Jahren 1985 und 1989 relevant, die zum ersten Mal explizit die „Verfolgungen und Massenvernichtungen“(BUNDESMINISTER FÜR UNTERRICHT, KUNST UND SPORT 1989:743) als Thema für den Geschichts- 
unterricht aufgriffen. Der Lehrplan für Geschichte an der AHS wurde anschließend in den Jahren 2004/2005, 2008, 2016 und 2018 geändert. Durch die Änderung im Jahr 2008 wurde das historische Lernen mit der politischen Bildung verknüpft, indem die Fachbezeichnung und die Inhalte von „Geschichte und Sozialkunde“ zu „Geschichte und Sozialkunde / Politische Bildung“ geändert wurden (BUNDESMINISTERIN FÜR UNTERRICHT, KUNST UND KULTUR 2008:10-15). Seit 2016 werden kompetenzorientierte Lehrpläne für das Fach „Geschichte und Sozialkunde / Politische Bildung“ für die Unter- und Oberstufe klassenweise aufsteigend eingeführt (vgl. BUNDESMINISTERIN FÜR BILDUNG UND FRAUEN 2016a und 2016b). In dem 2016 eingeführten Lehrplan für die AHS Unterstufe ist der Themenkomplex in der 4. Klasse im Modul „Faschismus - Nationalsozialismus - politische Diktaturen" angesiedelt (BUNDESMINISTERIN FÜR BILDUNG UND FRAUEN 2016a:22). Neben Kompetenzerwerb in den Bereichen Analyse bildlicher und schriftlicher Quellen, Perspektivität und Handlungskompetenz stehen inhaltlich folgende Punkte auf dem Lehrplan:

Ausgewählte Aspekte faschistischer bzw. diktatorischer Systeme im Europa des 20. Jahrhunderts vergleichen und Strukturmerkmale herausarbeiten (,Ständestaat/Austrofaschismus', italienischer Faschismus, Nationalsozialismus, Stalinismus, DDR); Grundlagen, Voraussetzungen und Auswirkungen des Nationalsozialismus in Österreich analysieren; Historische Alltagswelten in Demokratie und Diktatur vergleichen; Geschichtskulturelle Produkte (z.B.: Computerspiele oder Spielfilme) kritisch hinterfragen. (BUNDESMINISTERIN FÜR BILDUNG UND FRAUEN 2016a:22)

Das Thema Nationalsozialismus in Österreich und der damit zusammenhängende ,Anschluss“ wird hier in einen größeren Zusammenhang der vergleichenden Betrachtung verschiedener diktatorischer Systeme und ihrer Auswirkungen auf die Bevölkerung eingeordnet. Die Ausweitung und zugleich Eingrenzung ist dabei vor allem geographischer Natur: es sollen Diktaturen in Europa verglichen werden. Aus dem Unterpunkt „Historische Alltagswelten in Demokratie und Diktatur vergleichen" geht außerdem das Ziel der Demokratiebildung deutlich hervor (vgl. ALAVI / POPP 2012:7f.).

Für die AHS Oberstufe ist der Themenkomplex nochmals in der 7. Klasse im Kompetenzmodul 5 angesiedelt (vgl. BUNDESMINISTERIN FÜR BILDUNG 2016:57f.). Auf Kompetenzebene sollen hier unter anderem das eigenständige Formulieren von Fragen an die Vergangenheit, gattungsspezifische Quelleninterpretation, die Unterscheidung und Reflexion von Begriffen der Alltags- und Fachsprache sowie die Unterscheidung von Vorurteilen und Sachurteilen und die Berücksichtigung von Grundhaltungen Betroffener in historischen Prozessen gestärkt werden. Auf thematischer Ebene stehen folgende Bereiche im Lehrplan: 
Nationale und internationale Politik zwischen 1918 und 1945, z.B.: Friedensverträge, Krisen der Zwischenkriegszeit, Zweiter Weltkrieg; demokratische, autoritäre und totalitäre Staatensysteme und ihre Ideologien in Europa; Darstellung von Ideologien in geschichtskulturellen Produkten; nationalsozialistisches System und Holocaust; Erinnerungskulturen im Umgang mit dem Holocaust; das bipolare Weltsystem 1945-1990, sein Zusammenbruch und die Transformation des europäischen Systems. (BUNDESMINISTERIN FÜR BILDUNG 2016:57f.)

Auch hier wird das Thema Nationalsozialismus in einem größeren Gesamtzusammenhang verortet, der diesmal jedoch nicht geographisch, sondern chronologisch-epochal begrenzt wird. Das Kompetenzmodul 5 soll das 20. Jahrhundert als Epoche abbilden und macht damit gängige Periodisierungskonzepte der Geschichtswissenschaft sichtbar (vgl. RoTHFELS 1956; HOBSBAWM 1995).

An der Gegenüberstellung zeigt sich, dass der Nationalsozialismus zwar in der Oberstufe in einen größeren Kontext gesetzt wird, da die Zeitspanne des Kompetenzmoduls 5 von 1918 bis 1990 reicht, jedoch die Herangehensweise zum Umgang mit dem Thema in Unter- und Oberstufe grundsätzlich ähnlich ist.

\section{Kapitelstrukturen, Autorentexte und der Umgang mit dem ,Anschluss ${ }^{6}$}

Der sogenannte ,Anschluss' Österreichs wird in den Schulgeschichtsbüchern unterschiedlich eingebettet und thematisiert. Die Kette von Ereignissen wird entweder in den Kontext von Diktaturen in Europa oder in das Kapitel zur Ersten Republik eingeordnet. Durch die Einordnung in das Kapitel zu Diktaturen wird der Zusammenhang mit dem Nationalsozialismus stärker betont, während eine Einordnung in den Kontext der Ersten Republik die Verbindungen zum ,Austrofaschismus' stärker hervorhebt. Die Zuordnung des ,Anschlusses` zu einem Themenkomplex scheint dabei in der Gesamtschau nicht eindeutig. Die unterschiedlichen Zuordnungen können zu chronologischen Brüchen führen. So wird im Schulbuch Zeitbilder 4 der ,Anschluss“ unter der Überschrift „Das Ende der Ersten Republik“ erst im Modul 3 „Hundert Jahre Republik Österreich“ aufgegriffen (vgl. EBENHOCH / KRONBERGER-SCHMID / SCHEUCHER / WALD 2019:60f.). Die Module 1 und 2, die dem vorangestellt sind, beschäftigen sich aber bereits mit Diktaturen in Europa sowie Rassismus und Völkermord am Beispiel des Nationalsozialismus (vgl. EBENHOCH / KRONBERGER-SCHMID / SCHEUCHER / WALD 2019:631, 32-55.) - also mit Themen, die in chronologisch-ereignisgeschichtlicher Hinsicht in einem inhärenten Zusammenhang mit dem ,Anschluss` stehen. Ähnliches findet sich auch in anderen Schulbüchern (vgl. PAIREDER / HOFER 2019:27f.). Dies widerspricht dem, was HANS-JÜRGEN PANDEL (2005:21) für das Erzählen 
in Schulbüchern als „Vorgriffsverbot“ bezeichnet hat. Damit ist gemeint, dass die chronologische und kausale Abfolge von Ereignissen nicht durch Vorgriffe auf Themen, die im Unterricht erst später behandelt werden, durchbrochen werden sollte (vgl. PANDEL 2005:21). Solch chronologische Brüche in der Erzählstruktur verlangen von den Schülerinnen und Schülern viel Interpolationsleistung, um Kohärenz in der Erzählung herzustellen (vgl. PANDEL 2005:27-30). Aber nicht nur die Zuordnung des Ereignisses zu einzelnen Kapiteln, sondern auch die Kapitel- bzw. Unterkapitelüberschriften transportieren diskursive Zusammenhänge und Deutungen sowie geschichtsdidaktische Zugriffsweisen und Herausforderungen. Der Nationalsozialismus wird häufig zunächst als deutsches Phänomen charakterisiert. Nach der überwiegend ereignisgeschichtlichen Thematisierung des ,Anschlusses“ im März 1938 wird dann zwischen Deutschen und Österreicherinnen sowie Österreichern kein expliziter, aber teilweise durchaus ein impliziter Unterschied gemacht. Kapitelüberschriften wie „Deutschland: der totalitäre NS-Staat“" (GRAF / VOGEL-WALDHÜTTE / HALBARTSCHLAGER 2018:13) und „Nationalsozialistischer Terror in Österreich“ (MONYK / SCHREINER 2019:19) suggerieren durchaus eine implizite Unterscheidung, möglicherweise nach wie vor als eine Art Abgrenzungsmechanismus. Letzteres meint dabei den Versuch vieler Menschen in Österreich und Deutschland, sich von den Verbrechen des Nationalsozialismus abzugrenzen, um sich nicht mit eigener Schuld auseinandersetzen zu müssen. Durch die sogenannte ,Opferthese“ wurde dieser Mechanismus in Österreich auch politisch unterstützt.

In den Autorentexten wird der ,Anschluss ‘ hauptsächlich ereignisgeschichtlich als eine Kette von mehr oder minder zusammenhängenden Ereignissen erzählt, beginnend beim nationalsozialistischen Putschversuch und dem Tod von Engelbert Dollfuß im Jahre 1934, über das Zusammenrücken der Diktatoren Benito Mussolini und Adolf Hitler, Kurt Schuschniggs Besuch in Berchtesgaden, die von Schuschnigg für den 13. März anberaumte Volksbefragung, die dann aufgrund des Einmarschs der Wehrmacht am 12. März nicht stattfand bis schließlich hin zum „Gesetz über die Wiedervereinigung Österreichs mit dem Deutschen Reich“. Die Autorentexte sind dabei einerseits geprägt von einer auf einzelne Akteure fokussierten Erzählweise:

Hitler war fest entschlossen, Österreich an das Deutsche Reich anzuschließen. Im Februar 1938 befahl er Schuschnigg zu einer Unterredung nach Berchtesgaden. Als Ergebnis musste der österreichische Bundeskanzler den Nationalsozialisten Arthur Seyß-Inquart zum ,Sicherheitsminister' ernennen. Schuschnigg wollte dem weiteren nationalsozialistischen Druck durch eine Volksbefragung am 13. März 1938 begegnen. (STAUDINGER / EBENHOCH / SCHEUCHER / SCHEIPL 2019a:50) 
Andererseits finden sich auch Schulbücher, die den ,Anschluss' weniger akteursals vielmehr zeitpunktorientiert erzählen:

Je mächtiger Hitler wurde, umso größer wurde der Druck auf Österreich, sich mit Deutschland zu vereinigen. Um dem zuvorzukommen, wollte der damalige Bundeskanzler Schuschnigg die Bevölkerung abstimmen lassen [...]. Aus der Volksbefragung am 13. März wurde dann nichts, da bereits am 12. März deutsche Truppen in Österreich einmarschierten. Am 15. März erklärte Hitler am Heldenplatz in Wien den ,Anschluss“ Österreichs an das „Dritte Reich“, am 10. April wurde eine Volksabstimmung abgehalten. (GRAF/ VOGEL-WALDHÜTTE / HALBARTSCHLAGER 2018:25)

Beiden Auszügen ist gemein, dass sie Hitler als treibende Kraft ins Zentrum der Erzählung stellen. Eine Interpretation der Vergangenheit abseits der Fokussierung auf diese Einzelperson wird damit erschwert. Im Hinblick auf das geschichtswissenschaftliche und geschichtsdidaktische Grundkonzept der Narrativität findet sich hier das, was Hans-Jürgen Pandel als „Ereigniskonfetti“ (PANDEL 2017:101) in Schulbüchern beschrieben hat - also eine Aneinanderreihung von Ereignissen ohne sinnstiftende Verknüpfung. Überspitzt formuliert bedeutet das: Die Autorentexte zum ,Anschluss' sind zwar informationsgesättigte Texte, aber keine Narrationen (vgl. PANDEL 2005:15-30, insb. 31; BORRIES 2005:43).

Auf erinnerungskultureller Ebene zeigen die akteurs- und ereigniszentrierten Zugriffe der Autorentexte, welche Schritte zum ,Anschluss` als besonders wichtig erachtet werden: das Treffen in Berchtesgaden und Schuschniggs Bemühungen, durch eine Volksbefragung die Unabhängigkeit Österreichs zu sichern, der Einmarsch der Wehrmacht und die Volksabstimmung unter den Nationalsozialisten. Auffällig ist die positive Konnotation Schuschniggs, denn die Verknüpfung zu Ständestaat und ,Austrofaschismus' spielt im Zusammenhang mit dem ,Anschluss ${ }^{6}$ keine Rolle mehr.

\section{Konzeptsensibilität und Aufgabenstellungen}

In allen Schulbüchern wird der ,Anschluss` Österreichs mit Anführungszeichen als problembehafteter, da vielschichtig ideologisch aufgeladener Begriff gekennzeichnet (vgl. KLIMPFINGER 2018). Ein Schulbuch regt mit der Aufgabenstellung „Die NS-Propaganda bezeichnete den Einmarsch in Österreich als ,Anschluss'. Diskutiert in der Klasse, was mit dieser Wortwahl ausgedrückt wird!“ (MONYK / SCHREINER 2019:20) auch zu einer sprachlichen Reflexion dieses vielfach verwendeten Begriffs an, woran ein begriffssensibler Zugang deutlich wird. Ein ähnlicher Zugriff findet sich auch im Schulbuch Zeitbilder 4 mit der folgenden Aufgabenstellung: 
Auf diesen beiden Seiten sowie im gesamten folgenden Kapitel sind zahlreiche Begriffe unter Anführungszeichen gesetzt. Sammelt in Gruppenarbeit alle diese Begriffe, findet ihre Bedeutungen heraus und erstellt ein Lexikon. Hinterfragt dabei auch immer kritisch die Zusammenhänge, in denen diese Begriffe von den Nationalsozialisten verwendet wurden. (EBENHOCH / KRONBERGER-SCHMID / SCHEUCHER / WALD 2019:40)

Solche auf die Problembehaftung einzelner Begriffe ausgerichteten Zugriffe lassen sich gut mit der Theorie des konzeptsensiblen Geschichtsunterrichts verknüpfen, die im Folgenden vertiefend aufgegriffen werden soll. Konzeptsensibler Geschichtsunterricht meint dabei nach Peter Geiss „eine Form des historischen Lernens, die zentrale Konzepte sowohl auf der Ebene des Betrachteten (Quellentermini) als auch auf der Ebene der Betrachtung (Fachtermini der Geschichtswissenschaft) als problematisierungsbedürftig ansieht" (GEISS 2017:153). Das Schulbuch querdenken - Geschichte und politische Bildung 4 setzt konzeptsensible Zugänge um, indem in gesonderten Kästen „Basiskonzepte“ erklärt werden - auf der Seite mit der Überschrift „,Anschluss“ Österreichs an das Deutsche Reich“ (MADER / STRUTZ 2019:34) beispielsweise das Konzept von Zeitpunkten und ihrer Strukturierungsfunktion im Umgang mit Vergangenheit. Inwiefern diese Kästen nun aber im Unterricht verwendet werden und so tatsächlich konzeptsensibles historisches Lernen ermöglicht wird, bleibt an dieser Stelle offen. Ein grundsätzlich ähnlicher, aber auf einem komplexeren Niveau stattfindender Zugriff wurde im Schulbuch Zeitbilder 7/8 für die Oberstufe gewählt. Dort werden auf einer Doppelseite zum Kompetenztraining der historischen Sachkompetenz die Begriffe ,Faschismus' und ,Totalitarismus anhand von Autorentexten, Definitionen und einer Tabelle zum Vergleich der totalitären Systeme des Faschismus, Nationalsozialismus und Stalinismus vermittelt (vgl. StAUDingER / EBENHOCH / SCHEUCHER / SCHEIPL 2019b:30f.).

Was hierbei fehlt, ist eine Aufgabenstellung zur Bearbeitung der Doppelseite, sodass nicht klar wird, ob und wie in der Praxis der konzeptsensible Zugang tatsächlich umgesetzt wird. Weitere Aufgabenstellungen wie „Diskutieren Sie in einer Kleingruppe über die Mitverantwortung von ZuschauerInnen“ (MELICHAR / PLATTNER / RAUCHEGGER-FISCHER / SCHARINGER 2018:43) regen zu kritischhistorischem Denken und einem reflektierten Umgang mit Begriffen von Täterschaft an. Im Sinne der verstärkten Kompetenzorientierung und der Verschränkung von historischer und politischer Bildung in einem Schulfach wird diese Aufgabe als politische Handlungskompetenz gekennzeichnet. An dem Umgang mit revisionistischen Tendenzen spiegelt sich die in den Schulbüchern angestrebte Verknüpfung historischer und politischer Kompetenzen besonders deutlich wider. Das Schulbuch Zeitbilder 7 greift dieses Thema an mehreren 
Stellen auf: Unmittelbar auf die ereignisgeschichtliche Thematisierung des sogenannten ,Anschlusses “ folgt auf der nächsten Doppelseite im Unterkapitel „Das Hakenkreuz über Österreich“ (STAUDINGER / EBENHOCH / SCHEUCHER / SCHEIPL 2014:60) zunächst ein Abschnitt mit der Überschrift „Die sogenannten ,positiven“ Erinnerungen“ (STAUDINGER / EBENHOCH / SCHEUCHER / SCHEIPL 2014:60). In diesem Abschnitt werden der Geschichtsrevisionismus und eine positive Einstellung zum Nationalsozialismus durch Formulierungen wie: „Es gibt heute noch Menschen - oft ehemalige Nationalsozialisten oder deren durch das Elternhaus geprägte Kinder und Enkel -, die von ,positiven Seiten der nationalsozialistischen Diktatur in Österreich sprechen" (STAUDINGER / EBENHOCH / SCHEUCHER / SCHEIPL 2014:60) verurteilt und politisch kontextualisiert. Hinzugefügt wird außerdem ein Ausschnitt aus der Forschungsliteratur über die mentalitätsgeschichtliche Situation nach dem Zweiten Weltkrieg (vgl. RATHKOLB 2011:16) sowie eine Aufgabenstellung, die die Schülerinnen und Schüler auffordert, psychologische Erklärungsmuster für positive Erinnerungen an den Nationalsozialismus und Verdrängungsmechanismen zu suchen (vgl. STAUDINGER / EBENHOCH / SCHEUCHER / SCHEIPL 2014:60). Bemerkenswert hierbei: Diese Herangehensweise stammt aus der älteren Auflage des Schulbuchs. In der neuen Auflage wird der Themenkomplex deutlich geraffter dargestellt, zusammengefasst auf einer Doppelseite mit der Überschrift „Austrofaschistischer Ständestaat und sieben Jahre NS-Herrschaft" (STAUDINGER / EBENHOCH / SCHEUCHER / SCHEIPL 2019a:50). Auch dort gibt es einen kurzen Unterabschnitt mit dem Titel „Die ,positiven“ Erinnerungen an die NS-Zeit“" (STAUDINGER / EBENHOCH / SCHEUCHER / SCHEIPL 2019a:51), dieser ist jedoch weniger klar in der Deutung, Erklärung und Verurteilung solch revisionistischer Tendenzen, da die gerade zitierten Sätze, der Auszug aus der Forschungsliteratur sowie die Aufgabenstellung gestrichen wurden. Aus solchen Veränderungen in den Schulbüchern lässt sich für eine Bilanz zur Erinnerungskultur schließen, dass nach wie vor Uneinigkeit im Umgang mit revisionistischen Tendenzen besteht. Dieser Eindruck wird in den später folgenden Kapiteln jedoch ein wenig relativiert, denn hier werden im Kontext des Holocaust die ,Auschwitzlüge“ und die ,Mauthausenlüge" aufgegriffen und zum Basiswissen zu diesem Kapitel gehört der Grundbegriff ,Revisionismus' (STAUDINGER / EBENHOCH / SCHEUCHER / SCHEIPL 2019a:75 u. 87). Eine Aufgabenstellung fordert die Schülerinnen und Schüler zu einer näheren Auseinandersetzung mit diesem Themenkomplex der Erinnerungskultur auf: „Erkläre die Begriffe ,Auschwitzlüge‘, ,Mauthausenlüge“, Revisionismus“ (STAUDINGER / EBENHOCH / SCHEUCHER / SCHEIPL 2019a:75). Dies macht vor allem Aspekte der Demokratie- und Werteerziehung deutlich. 
Für eine Bilanz zur Erinnerungskultur bedeutet dieser Befund aber auch, dass eine gegenwärtige Notwendigkeit gesehen wird, diese Aspekte so explizit zu thematisieren und zu bewerten; möglicherweise auch, um gegenwärtigen geschichtsrevisionistischen Tendenzen entgegenzuwirken.

In diesen Kontext einzuordnen ist auch eine Art ,Nicht-Befund'. Die sogenannte ,Opferthese', die in der offiziellen österreichischen Erinnerungskultur und vor allem der Geschichtspolitik lange Zeit vorherrschend war, kommt in den Schulbüchern selten zur Sprache - weder wird sie vertreten noch - und das ist vielleicht der interessantere Befund - wird sie in den Abschnitten, die sich dezidiert mit Erinnerungskultur beschäftigen als historisch-erinnerungskulturelles Phänomen aufgegriffen. Zwei Ausnahmen finden sich jedoch, eine bildet das Schulbuch MEHRfach Geschichte 4, in dem es heißt: „Österreich als Opfer? Nach 1945 wurde Österreich oft als erstes Opfer Nazi-Deutschlands bezeichnet, obwohl viele Menschen den ,Anschluss' 1938 begrüßt hatten. Die Opfertheorie wurde erst Jahrzehnte später hinterfragt" (GRAF / VOGEL-WALDHÜTTE / HALBARTSCHLAGER 2018:25). Die andere Ausnahme stellt das Schulbuch Zeitbilder 4 dar, das das Thema im Rahmen der Doppelseite „Die Zweite Republik - ein neues Österreich“ aufgreift und die Opferthese auch anhand der Moskauer Deklaration und der Frage nach Entnazifizierung umfassend thematisiert (vgl. EBENHOCH / KRONBERGERSCHMID / SCHEUCHER / WALD 2019:134f.). Eine Historisierung des staatlichen und gesellschaftlichen Umgangs mit der NS-Zeit in der Zeit danach erfolgt also in Ansätzen, jedoch wird der ,Opfermythos' noch nicht vollumfänglich als erinnerungskulturelles und geschichtspolitisches Phänomen aufgegriffen.

Nimmt man weniger die inhaltliche als vielmehr die didaktische Dimension der Aufgabenstellungen hinsichtlich geschichtsdidaktischer Grundprinzipien in den Blick, so zeigt sich ein gemischtes Bild. Einige Schulbücher setzen das geschichtsdidaktische Prinzip der Multiperspektivität deutlich um, beispielsweise indem mit verschiedenen Zeitzeugenberichten gearbeitet wird: „Vergleiche die drei Zeitzeugenberichte zum ,Anschluss` Österreichs. Arbeite Gemeinsamkeiten und Unterschiede heraus. Erörtere mögliche Gründe für die unterschiedlichen Wahrnehmungen“"(vgl. EBENHOCH / KRONBERGER-SCHMID / SCHEUCHER / WALD 2019:18f.). Andere Schulbücher stellen die Quellenkritik als Methode bei den Aufgabenstellungen in den Vordergrund: „Beurteile und vergleiche die folgenden Materialien: Welche Art von Material hast du vor dir? Aus welcher Zeit stammt es? Welches Österreichbild wird präsentiert? Welchen Wert hat es für jemanden, der sich mit diesem Ereignis der österreichischen Vergangenheit befasst?“" (PAIREDER / HOFER 2019:28) Viele Schulbücher fokussieren hinsichtlich des ,Anschlusses“ vor allem reproduktive Aufgabenstellungen, die zum Teil recht 
umfangreiche Textproduktion und viel Vorwissen von den Schülerinnen und Schülern erwarten: „Fasse die wesentlichen Auswirkungen des Nationalsozialismus auf Österreich mit eigenen Worten zusammen!“ (MONYK / SCHREINER 2019:20) Ob diese Aufgabenstellung einzig auf Basis der Informationen auf den entsprechenden Schulbuchdoppelseiten voll ständig bearbeitet werden kann, ist dabei durchaus in Frage zu stellen.

\section{Mentalitätsgeschichtliche Aspekte und Deutungsmuster}

In manchen Schulbüchern erfolgt neben dem ereignisgeschichtlichen Zugriff auch eine mentalitätsgeschichtliche Einordnung, die in der Deutungsebene jedoch unterschiedlich ausfällt. Einige Schulbücher nutzen Aufgabenstellungen, die im Sinne von problemorientiertem, selbstständigem Lernen die Schülerinnen und Schüler dazu auffordern, die Mentalität in Österreich im Kontext des ,Anschlusses anhand der Volksabstimmung am 10. April 1938 zu reflektieren:

Erkläre, warum das Abstimmungsergebnis nicht repräsentativ für die allgemeine Meinung in Österreich war. Überlege, warum so viele Menschen für den ,Anschluss ${ }^{6}$ gestimmt haben. Nenne politische, wirtschaftliche und private Gründe. (GRAF / VOGEL-WALDHÜTTE / HALBARTSCHLAGER 2018:30, Herv. im Original)

Auf der anderen Seite findet sich in einem Autorentext aber auch dieser Abschnitt: „15. März 1938: Hitler traf in Wien ein. Hunderttausende jubelten ihm zu. Doch viele Österreicher und Österreicherinnen waren auch besorgt und trauerten um die Unabhängigkeit ihres Landes“" (MONYK / SCHREINER 2019:20). Zwar wird eine positive Mentalität im Zuge von Hitlers Aufenthalt in Wien im März 1938 nicht verschwiegen, aber die Formulierung dieses Abschnittes legt den Fokus doch ganz klar auf den letzten Satz und damit auf eine ablehnende Haltung dem ,Anschluss' gegenüber. Dieser Eindruck wird erzeugt durch den kurzen, unpräzisen Satz zum Jubel im Gegensatz zu der präzisen Benennung „,viele Österreicherinnen und Österreicher". Ein solcher Mechanismus, Beteiligung und Begeisterung zwar zu thematisieren, anhand der Gewichtung und durch sprachliche Strukturen jedoch trotzdem eine Deutung in Richtung apologetischer Tendenzen zu transportieren, findet sich auch in anderen Schulbüchern (vgl. EngBerding / HeIHSLER / PORSTNER / WURZRAINER 2019:29). Für eine Bilanz zur österreichischen Erinnerungskultur am Beispiel von Schulbüchern bedeutet dies, dass - zumindest teilweise - nach wie vor verschleiernde Mechanismen greifen, obwohl die historische Forschung sich inzwischen über eine Beteiligung von Österreicherinnen und Österreichern an Verbrechen des Nationalsozialismus einerseits (vgl. DOKUMENTATIONSARCHIV DES ÖSTERREICHISCHEN 
WIDERSTANDES 2014) und über die in weiten Teilen zustimmende Haltung der Bevölkerung (vgl. KERSHAW 2016:449; BENZ 2010:158-160) nach dem ,Anschluss“ andererseits einig ist. Für eine Bilanz zur Erinnerungskultur ist weiterführend auch die Frage zentral, wie der Themenkomplex Holocaust eingebunden wird und auch wie mit der Beteiligung von Österreicherinnen und Österreichern an den Verbrechen des Holocaust umgegangen wird. Die von Christoph Kühberger und Herbert Neureiter vorgetragenen Überlegungen zum geschichtsdidaktischen Umgang mit dem Holocaust, die im Rahmen einer empirischen Studie angestellt wurden, spiegeln sich dabei in den Schulbüchern wider. Kühberger und Neureiter konstatieren, dass sich der schulisch-geschichtsdidaktische Umgang mit diesem Thema heute durch eine „Verzahnung der historischen und politischen Bildung“" (KÜHBERGER / NEUREITER 2017:15) und durch „Befreiung von Emotionalisierung und Überwältigung zugunsten einer Anregung des kritisch historischen Denkens auszeichne“" (KÜHBERGER / NEUREITER 2017:15f.). In den hier betrachteten Schulbüchern werden die Grauen des Holocaust zwar angesprochen, ohne dabei jedoch - vor allem auf bildlicher Ebene - eine emotionale Überwältigung hervorzurufen. Fragen nach Täterschaft und Verantwortung werden mal implizit, mal explizit gestellt. Ein Schulbuch behandelt das Thema Holocaust als Unterthema im Kapitel „Der Nationalsozialismus in Deutschland“ (BRZOBOHATY / KOWARZ / SALMEYER / ZELLHOFER 2013:39) und legt damit den Fokus auf deutsche Täterschaft. Dies kann in der Traditionslinie des ,Opfermythos' verstanden werden (vgl. HAMMERSTEIN 2017:57-67). Ein anderes Schulbuch greift dagegen die Frage nach Täterschaft explizit auf, indem auf einer Seite der Themenkomplex ,Täterschaft ${ }^{\star}$ als Frage der Forschung und der Erinnerungskultur aufgegriffen wird (vgl. MELICHAR / PLATTNER / RAUCHEGGER-FISCHER / SCHARINGER 2018:43).

Uneinig sind sich die Schulbücher in ihren Deutungsmustern weiterhin darüber, wann die Demokratie in Österreich endete. Einige Schulbücher machen deutlich, dass die Demokratie der Ersten Republik 1934 ein vorzeitiges Ende fand, indem sie das Dollfuß-Regime, den Bürgerkrieg und den Austrofaschismus thematisieren. Im Band Zeitbilder 7/8 wird beispielsweise die Kanzlerschaft von Engelbert Dollfuß unter der Überschrift „Das Ende der Demokratie“ (SCHEIPL / SCHEUCHER / EBENHOCH / STAUDINGER 2019:46) vermittelt. Andere Schulbücher wiederum sehen den 12. März 1938 als „Ende der Ersten Republik“ (PAIREDER / HOFER 2019:28). Hieran zeigt sich eine diskursive Ambivalenz hinsichtlich der Deutung des Dollfuß-Schuschnigg-Regimes wie auch eine Uneinigkeit im Demokratieverständnis. Für das historische Lernen und die Herausbildung eines ,demokratischen Geschichtsbewusstseins“ (BORRIES 2007:211f.) könnten diese Differenzen genutzt werden, um einerseits Demokratie als Phänomen zu historisieren 
und anderseits zu hinterfragen, inwiefern gegenwärtige und zeitgenössische Demokratieverständnisse Einfluss auf die Darstellung von Vergangenheit haben. Diese Perspektive wird von den Schulbüchern selbst jedoch nicht angeregt, da diese immer nur eine Seite vermitteln.

\section{Quellenkanon und Symbolbilder?}

Der Umgang mit Schriftquellen unterscheidet sich in den Schulbüchern deutlich. Naturgemäß ist vor allem ein unterschiedlicher Umgang in den Büchern der Unterstufe im Vergleich zur Oberstufe zu bemerken, was mit der stärkeren Ausrichtung des Geschichtsunterrichts der Oberstufe auf Kompetenzen der Quellenanalyse zusammenhängt. Der Lehrplan für die AHS-Unterstufe schreibt für die 4. Klasse im Modul 1 jedoch auch schon konkrete Kompetenzen in der Quellenarbeit vor: ,Schriftliche und bildliche Quellen beschreiben, analysieren und interpretieren; Perspektivität von Quellen wahrnehmen; Erkenntnisse aus Quellenarbeit oder Arbeit mit Darstellungen für individuelle Orientierung nutzen“ (BUNDESMINISTERIN FÜR BILDUNG UND FRAUEN 2016a:22). Die Art und Weise, wie Schulbücher Quellen für den inhaltlichen Bereich des ,Anschlusses ‘ und des Nationalsozialismus in Österreich bereitstellen, ist dabei jedoch durchaus unterschiedlich. So findet sich im Schulbuch Geschichte für alle 4 beispielsweise ein kurzer Quellenauszug in den Autorentext eingeschoben, mit der Überschrift „Unterredung zwischen Hitler und Schuschnigg in Berchtesgaden am 12.02.1938“ (MONYK / SCHREINER / MANN 2010:44). Weitere Angaben zur Herkunft der Quelle, zum Autor und zum Entstehungskontext werden nicht gegeben. Der nachfolgend abgedruckte Dialog stammt aus den Erinnerungen Kurt Schuschniggs Ein Requiem in Rot-Weiß-Rot und ist in der Darbietung im Schulbuch stark gekürzt und teilweise sprachlich verändert (vgl. SCHUSCHNIGG 1978:38-44), ohne dass diese Kürzungen und Veränderungen kenntlich gemacht werden. Obwohl dieser Text in der Forschung als „Schlüsseldokument der österreichischen Geschichte“ (BAUER 2017:22) bezeichnet wird, wird er in den Schulbüchern kaum als Quelle verwendet.

Aus didaktischer Perspektive ist auch die Anordnung und Relation von Quellen und Autorentexten interessant. Die meisten Schulbücher präsentieren Autorentexte, Quellentexte, Bilder und Aufgabenstellungen auf einer (Doppel-)Seite, wobei die unterschiedlichen Textgattungen meist durch Layout und farbliche Hinterlegungen voneinander abgegrenzt werden. Einige Schulbücher lösen jedoch Quellen und Aufgabenstellungen in separaten Übungsteilen vom Autorentext (vgl. MADER / STRUTZ 2019:46), sodass von den Schülerinnen und Schülern eine Ver- 
knüpfungsleistung verlangt wird. Dies erschwert die zu erbringenden Denkleistungen und lässt den Autorentext als interpretative Leitlinie erscheinen, die durch die Quellen nur unterstützt wird.

Ein Aspekt, der im Kontext des ,Anschlusses“ häufiger aufgegriffen wird, ist die Volksabstimmung, die die Nationalsozialisten am 10. April 1938 durchführten. Dieser Zusammenhang wird in den Schulbüchern als Anlass zur Demokratiebildung genommen, indem der Stimmzettel dieser Abstimmung als Quelle thematisiert wird. Der Aspekt der Demokratieerziehung tritt dann in den zugehörigen Aufgabenstellungen sehr explizit zu Tage: „Analysiere den Stimmzettel. Begründe, warum so ein Stimmzettel in Demokratien nicht eingesetzt werden darf“" (ENGBERDing / HeIHSLER / PORSTNER / WURZRAINER 2019:46).

Neben textuellen Medien, die sich in Schulbüchern in vielfältiger Ausprägung von Autorentexten über Quellen bis zu Aufgabenstellung finden, stehen visuelle Medien unter anderem in Form von Bildern, aber auch Karten und Karikaturen. Bilder haben im Schulbuch als Medium des historischen Lernens eine zentrale Bedeutung, ,da sie nicht nur einen wichtigen Anteil an der public memory einnehmen, sondern die starke Prägekraft visualisierter Geschichte auch für die Entwicklung eines individuellen und kollektiven Geschichtsbewusstseins als Voraussetzung angesehen wird“ (MITTNIK 2017:121f.). Dabei lassen sich verschiedene visuelle Bildtypen unterscheiden. Der häufigste Bildtypus im hier betrachteten Zusammenhang ist der der Fotografie (vgl. MITTNIK 2017:122; weiterführend MARKOVA 2015b). Daneben stehen Plakate als beliebtes Medium. Die Verwendung von Bildern spiegelt in Teilen auch das wider, was Ina Markova mit einer größeren Stichprobe und einer diachronen Betrachtung im Rahmen einer Diskursanalyse von Bildtypen zur NS-Zeit in österreichischen Geschichtsschulbüchern bereits herausgearbeitet hat. Teilweise lassen sich in den aktuellen Schulbüchern aber auch Differenzen im Bildeinsatz herausarbeiten. So ist der These, dass Bilder des „Heldenplatz-Jubel[s] am 15. März 1938“ (MARKOVA 2015b:125) häufig durch Text-Bild-Relationen mit entschuldigenden oder herunterspielenden Deutungsmustern durch Verweise auf die NS-Propaganda in Verbindung stünden, welche österreichische Mittäterschaft und ideologische Überzeugung kaschieren oder ausklammern sollten (vgl. MARKOVA 2015b:125f.), nicht mehr vollumfänglich zuzustimmen. Sofern Bilder dieses Bildtyps vorkommen, der seit den 1970er Jahren Bestandteil des Bildkanons in Schulbüchern ist (vgl. MARKOVA 2015b:125), sind sie eingebettet in Narrationen, die eine ideologische Überzeugung von Österreicherinnen und Österreichern im Kontext des Jubels am Heldenplatz offen thematisieren. So beispielsweise im Band Zeitbilder 4 (EBENHOCH / KRONBERGER-SCHMID / SCHEUCHER / WALD 2019:18), in dem ein 
Bild vom Jubel auf dem Heldenplatz zu sehen ist. Dieses wird zwar als Propagandafoto ausgewiesen, jedoch nicht im Sinne der verschleiernden Mechanismen, die Markova herausgearbeitet hat. Vielmehr wird das Bild durch den Autorentext sowie drei Zeitzeugenerinnerungen als Quellen zusätzlich kontextualisiert und die Beteiligung und Begeisterung von Österreicherinnen und Österreichern beim ,Anschluss' offen dargelegt. In dem korrespondierenden Oberstufen-Band aus der Reihe Zeitbilder wird die (Mit-)Täterschaft von Österreicherinnen und Österreichern in der Ausgrenzung und Verfolgung von Jüdinnen und Juden durch ein Bild des Pressefotografen Albert Hilscher offengelegt, das zeigt, wie ein Kind unter den Augen eines Mannes mit Hakenkreuzbinde am Arm „Jud“ an eine Hauswand schreibt (vgl. STAUDINGER / EBENHOCH / SCHEUCHER / SCHEIPL 2019:51).

Laut Philipp Mittnik steht im Hinblick auf Bilder aus dem Kontext des Nationalsozialismus in Schulbüchern vor allem die Täterperspektive im Vordergrund (vgl. MitTNIK 2017:123, quantitative empirische Befunde 125-128). Die Bilder perpetuieren dabei die fotographische Ästhetik der Nationalsozialisten, ohne dass die Schulbücher zur kritischen Reflexion derselben einladen. Weiterhin fehlen häufig ergänzende Erläuterungen zum genauen Entstehungskontext der Fotografien, zur Überlieferungssituation sowie zur Beschränkung der Bilder auf einen Kanon aufgrund von Bildrechten und Lizenzen. Dies ist aus geschichtsdidaktischer Perspektive deutlich zu kritisieren, da es nicht dem im Geschichtsunterricht zu vermittelnden quellenkritischen Umgang mit Zeugnissen der Vergangenheit entspricht. Hinsichtlich einer Bilanz zur Erinnerungskultur anhand von Schulbüchern bedeuten diese Befunde, dass das visuelle Gedächtnis zum ,Anschluss' und zum Nationalsozialismus von der Perspektive der Täter geprägt ist. Eine Ausnahme bildet hier das Schulbuch Meine Geschichte 4 für die Unterstufe, das auf einer Seite Bilder als Propagandawerkzeug in Diktaturen anhand von vier Beispielbildern aus dem Nationalsozialismus mit ergänzenden Aufgabenstellungen thematisiert und so bei Schülerinnen und Schülern ein Bewusstsein für die Art und Weise, wie Bilder in einem diktatorischen System verwendet wurden, schaffen soll (vgl. PAIREDER / HOFER 2019:18). Ähnliches findet sich, wenn auch in weniger deutlich auf den Konstruktcharakter von Propagandafotos ausgerichteter Form, in einem anderen Schulbuch mit Blick speziell auf ein Bild vom Jubel am Heldenplatz, zu dem die Aufgabe gestellt wird „Beschreibe das Foto vom 15. März 1938. Schildere deinen Eindruck, den dieses Foto vermittelt" (EBENHOCH / KRONBERGER-SCHMID / SCHEUCHER / WALD 2019:18). Durch diese Aufgabenstellung können sowohl das Bild selbst als auch der Jubel auf dem Heldenplatz durch die Schülerinnen und Schüler kritisch hinterfragt werden. 
Im Hinblick auf den Umgang mit dem Holocaust in der Bildverwendung konstatierte Markova, es gäbe „kein anderes Motiv, das den Holocaust stärker vergegenwärtig[e], als Gleise, Waggons und Rampen“ (MARKOVA 2015b:129). Obwohl dies zweifelsohne stimmt - man denke an das Bild der Gleise, die nach Auschwitz führen - findet sich in den aktuellen österreichischen Schulbüchern ein weiteres Motiv, dass gerade für den österreichischen Kontext in ähnlicher, wenn vielleicht auch nicht ganz so ausgeprägter Form zum Symbolbild geworden ist: das Bild der sogenannten ,Todesstiege von Mauthausen“ (vgl. Melichar / PlatTNer / RAUCHEGGER-Fischer / SCHARINGER 2018:42; Brzobohaty / Kowarz / SALMEYeR / Zellhofer 2013:49). Das Konzentrationslager Mauthausen dient in der österreichischen Erinnerungskultur als „Kontrast- und Negativsymbol zur österreichischen Demokratie, und es dient wie kein anderer Erinnerungsort zur Veranschaulichung der Konsequenzen undemokratischer, totalitärer Entwicklungen“ (BOTZ / ELLMAUER / PRENNINGER 1998:20). Als geschichtspolitisch bedeutsamer Erinnerungsort ist Mauthausen auch in den Schulbüchern präsent - und das nicht nur auf bildlicher Ebene. Auch in den Autorentexten wird Mauthausen im Kontext des Holocausts aufgegriffen. Das Schulbuch Zeitbilder 7 geht sogar noch einen Schritt weiter und thematisiert im Text neben der sogenannten ,Auschwitzlüge‘ auch ausführlich die ,Mauthausenlüge', um so eine ganz klare Deutungslinie gegen revisionistische Tendenzen und Holocaustleugnung zu vermitteln.

\section{Geschichtspolitischer ,Minimalkonsens ${ }^{6}$}

Bezogen auf die geschichtspolitische Funktion von Schulgeschichtsbüchern sind die Seiten besonders interessant, die unter Überschriften wie „Basiswissen“ das gesamte Kapitel zusammenfassen. Neben der didaktischen Funktion solcher Seiten zur Wiederholung, als Referenzpunkt zum Nachschlagen und zur Prüfungsvorbereitung lässt sich hier auch eine geschichtspolitische Komponente ableiten: Diese Seiten beinhalten das, was als geschichtspolitischer ,Minimalkonsens' angesehen werden kann - das, was Schülerinnen und Schüler nach der Bearbeitung des Kapitels unbedingt wissen sollten. Nicht alle Schulgeschichtsbücher beinhalten diese zusammenfassenden Doppelseiten am Ende des Kapitels, exemplarisch sei hier nur auf eine solche Zusammenfassung hingewiesen (vgl. STAUDINGER / EBENHOCH / SCHEUCHER / SCHEIPL 2019:52f.).

Schülerinnen und Schüler sollen nach der Bearbeitung des Kapitels auf ereignisgeschichtlicher Ebene die Maiverfassung 1934, den Juliputsch mit der Er- 
mordung Dollfuß' im selben Jahr, die Kanzlerschaft Schuschniggs, das Juliabkommen 1936, die von Schuschnigg angesetzte Volksbefragung, den Einmarsch der Wehrmacht und die unmittelbar einsetzenden Verfolgungen kennenlernen. Auf der Ebene der Deutungen werden ihnen dann folgende Elemente mitgegeben: Schuschniggs Kanzlerschaft sei ,geprägt vom Ringen um Österreichs Unabhängigkeit" (STAUDINGER / EBENHOCH / SCHEUCHER / SCHEIPL 2019:52) - also durchaus positiv konnotiert, die Volksabstimmung hätte es den Bürgerinnen und Bürgern ermöglichen sollen, selbst über ihre Freiheit zu entscheiden, der sogenannte ,Anschluss' ist unmittelbar mit dem Einsetzen von Verfolgungen gleichzusetzen und Rassismus und Ausgrenzung hätten auch erst mit dem sogenannten ,Anschluss` begonnen. Die Mentalität in Österreich im März 1938, die im Kapitel zuvor durch den Auszug aus der Forschungsliteratur und das Bild vom Jubel auf dem Heldenplatz aufgegriffen wird, ist hier nicht Teil des geschichtspolitischen ,Minimalkonsenses ${ }^{6}$ - ebenso wenig Fragen von Täterund Mittäterschaft. Auch die Tatsachen, dass viele der in den entsprechenden Kapiteln und auch diesem Abschnitt zum Basiswissen genannten Opfergruppen in Österreich auch nach dem Ende des Zweiten Weltkrieges weiterhin ausgegrenzt wurden, ist nicht Teil des als grundlegend erachteten Wissens (vgl. UHL 2004).

\section{Fazit}

Schulgeschichtsbücher stellen als hochgradig geschichtspolitisch aufgeladenes Medium einen wichtigen Bestandteil der Erinnerungskultur dar. In ihnen manifestieren sich ein staatlich befürwortetes und gewolltes Bild der eigenen Nationalgeschichte und die Vorstellungen der Gegenwart von der Vergangenheit. Für die österreichische Erinnerungskultur im Hinblick auf 1938 und die folgenden sieben Jahre bedeutet dies konkret: Der Umgang mit dem 12. März 1938, aber auch den Ereignissen davor und danach ist in der Gesamtschau sehr ambivalent. Der ,Austrofaschismus' wird nicht immer im Zusammenhang mit dem Nationalsozialismus thematisiert. Ebenso wird Schuschnigg nicht immer im Zusammenhang mit dem Austrofaschismus thematisiert, sondern vielmehr als jemand charakterisiert, der um die Rettung Österreichs bemüht war. Die durchaus zustimmende Haltung vieler Österreicherinnen und Österreicher, die in der Forschung ausführlich herausgearbeitet wurde, wird in den Schulbüchern meist nur en passant thematisiert. Ebenso divergiert der Umgang mit Täter- und Mittäterschaft. Die Spanne reicht hier von der expliziten Thematisierung bis hin zum vorsichtigen Umgehen der Frage nach Täterschaft von Österreicherinnen und Österreichern. 
Gleichzeitig werden der ,Anschluss“ und die folgende Zeit des Nationalsozialismus in Österreich aber immer wieder explizit als Anlass zur gegenwartsbezogenen Demokratieerziehung genutzt. Unterschiede in der inhaltlichen Zuordnung des Ereignisses ,Anschluss', der Begrifflichkeiten und den didaktischen $\mathrm{Zu}$ gängen zeigen, dass die gegenwärtige Bewertung dieses Ereignisses in seiner Relevanz und seiner Deutung nicht einheitlich sind. Es scheint kein einheitlichkonsensfähiges Narrativ über den ,Anschluss' Österreichs zu geben. Zwar haben die Schulbücher den Anspruch, jeweils in sich ein geschlossenes Narrativ zu vermitteln - der Vergleich mehrerer Schulbücher zeigt aber Diskrepanzen, die auf eine diskursive Ambivalenz schließen lassen. Auf inhaltlicher Ebene wird dies beispielsweise an der Frage deutlich, wie mit dem Jubel auf dem Heldenplatz und der durchaus zustimmenden Mentalität vieler Österreicherinnen und Österreicher umgegangen wird. Dabei wirken Tendenzen der ,Opferthese “ immer noch nach. Ein Schritt zur Überwindung dieser diskursiven Ambivalenz könnte eine Thematisierung des ,Opfermythos' als geschichtspolitisches Phänomen im Schulbuch und damit eine Historisierung der Erinnerungskultur sein. Dominierende Tendenzen hinsichtlich der Darstellung des ,Anschlusses“ in aktuellen österreichischen Schulgeschichtsbüchern lassen sich kaum herausarbeiten, da die Gesamtschau ein ambivalentes Bild zeigt, dass sich zwischen vertiefter, konzeptsensibler Auseinandersetzung mit den historischen Ereignissen sowie der zugehörigen Erinnerungskultur und eher oberflächlicher Aneinanderreihung von Fakten bewegt. Die Art und Weise, wie die Ereignisse des Jahres 1938 in der Schulpraxis vermittelt werden, war nicht Teil dieses Beitrages, aber gleichwohl lohnen sich Untersuchungen in diese Richtung, wie sie an anderer Stelle zum Teil bereits angestellt wurden (vgl. KÜHBERGER / NEUREITER 2017).

\section{Literatur}

Alavi, Bettina / Popp, Susanne (2012): Menschenrechtsbildung - Holocaust Education-Demokratieerziehung. Einführung in den Themenschwerpunkt. In: Zeitschrift für Geschichtsdidaktik 11:7-10.

BAUER, KURT (2017): Die dunklen Jahre. Politik und Alltag im nationalsozialistischen Österreich 1933 bis 1945. Frankfurt a.M.

Becher, Ursula A. J. ('2017): Schulbuch. In: PANDEl, HANS-JÜRgen / SCHNeIDER, GERHARD (eds.): Handbuch Medien im Geschichtsunterricht. Schwalbach/Ts., 45-68.

BECKER, MANUEL (2014): Geschichtspolitik als Thema der Politikwissenschaft-Begriffsgeschichte, Forschungsstand, systematische Funktionszusammenhänge. In: Jahrbuch Extremismus \& Demokratie 26:51-69. 
Benz, Wolfgang (2010): Geschichte des Dritten Reiches. Bonn (= Schriftenreihe 377). BORRIES, BODO VON (1995a): Das Geschichtsbewußtsein Jugendlicher. Erste repräsentative Untersuchung über Vergangenheitsdeutungen, Gegenwartswahrnehmungen und Zukunftserwartungen von Schülerinnen und Schülern in Ost- und Westdeutschland. Weinheim (= Jugendforschung).

BORRIES, BODO VON (1995b): Das Geschichts-Schulbuch in Schüler- und Lehrersicht. Einige empirische Befunde. In: Internationale Schulbuchforschung 17:45-60.

BORRIES, BODO VON (2005): Schulbuch-Gestaltung und Schulbuch-Benutzung im Fach Geschichte. Zwischen empirischen Befunden und normativen Überlegungen. In: HANDRO, SASKIA / SCHÖNEMANN, BERND (eds.): Geschichtsdidaktische Schulbuchforschung. Berlin, 39-51. (=Zeitgeschichte-Zeitverständnis; Bd. 16).

BORRIES, BODO VON (2007): Demokratisches Geschichtsbewusstsein - Was könnte das sein und wie sollte es gefördert werden?. In: LANGE, DIRK / HIMMELMANN, GERHARD (eds.): Demokratiebewusstsein. Interdisziplinäre Annäherungen an ein zentrales Thema der Politischen Bildung. Wiesbaden, 209-228.

Botz, Gerhard / Ellmauer, Daniela / Prenninger, Alexander (1998): Mauthausen als „Erinnerungsort“. Probleme der „Authentizität“ und des österreichischen ,kollektiven Gedächtnisses “. In: Dokumentationsarchiv des österreichischen Widerstandes - Jahrbuch:15-29.

Brzobohaty, Johannes / Kowarz, Andreas / Salmeyer, Robert / Zellhofer, CHRISTA (2013): Zeitfenster 7. Wien.

BundESMINISTER FÜR UNTERRICHT, KunST UND SPORT (1989): Verordnung Änderung der Lehrpläne der allgemeinbildenden höheren Schulen. In: Bundesgesetzblatt für die Republik Österreich II, 63:623-968: https://www.ris.bka.gv.at/Dokumente/BgblPdf/1989 _63_0/1989_63_0.pdf(16.09.2019).

BundESMINISTERIN FÜR UNTERRICHT, KUnST UND KULTUR (2008): Änderung der Verordnung über die Lehrpläne der Volksschule, der Sonderschulen, der Hauptschulen und der allgemein bildenden höheren Schulen. In: Bundesgesetzblatt für die Republik Österreich II, 290: https://www.ris.bka.gv.at/Dokumente/BgblAuth/BGBLA_2008_II_290 /BGBLA_2008_II_290.pdfsig (16.09.2019).

BundeSMINISTERIN FÜR BILDUNG Und FRAuEN (2016a): Änderung der Verordnung über die Lehrpläne der Hauptschulen, der Verordnung über die Lehrpläne der Neuen Mittelschulen sowie der Verordnung über die Lehrpläne der allgemein bildenden höheren Schulen. In: Bundesgesetzblatt für die Republik Österreich II, 113: https://www.ris.bka.gv.at /Dokumente/BgblAuth/BGBLA_2016_II_113/BGBLA_2016_II_113.pdfsigl (07.08.2019).

BUNDESMINISTERIN FÜR BILDUNG (2016b): Änderung der Verordnung über die Lehrpläne der allgemein bildenden höheren Schulen; Änderung der Bekanntmachung der Lehrpläne für den Religionsunterricht an diesen Schulen. In: Bundesgesetzblatt für die Republik Österreich II, 219: https://www.ris.bka.gv.at/Dokumente/BgblAuth/BGBLA _2016_II_219/BGBLA_2016_II_219.pdfsig (24.03.2020). 
BUNDESMINISTER FÜR BILDUNG, WISSENSCHAFT UND FORSCHUNG (2018): Änderung der Verordnung über die Lehrpläne der allgemeinbildenden höheren Schulen; Änderung der Bekanntmachung der Lehrpläne für den Religionsunterricht sowie Bekanntmachung der Lehrpläne für den Religionsunterricht. In: Bundesgesetzblatt für die Republik Österreich II, 216: https://www.ris.bka.gv.at/Dokumente/BgblAuth/BGBLA_2018 II_216/BGBLA_2018_II_216.pdfsig (16.09.2019).

DOKUMENTATIONSARCHIV DES ÖSTERREICHISCHEN WIDERSTANDES (ed.) (2014): Täter. Österreichische Akteure im Nationalsozialismus. Wien (= Jahrbuch).

Ebenhoch, Ulrike / Kronberger-Schmid, Barbara / Scheucher, Alois / Wald, Anton (2019): Zeitbilder 4. Geschichte, Sozialkunde und Politische Bildung. Wien.

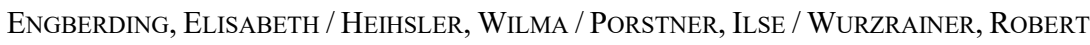
(2019): überall Geschichte 4. Geschichte und Sozialkunde / Politische Bildung. Wien.

GEISS, PETER (2017): Das Selbstbestimmungsrecht der Völker und seine Grenzen. Konzeptsensibler Geschichtsunterricht am Beispiel der Pariser Friedensordnung von 1919/20. In: Geiss, Peter / Heuser, Peter Arnold (eds.): Friedensordnungen in geschichtsdidaktischer und geschichtswissenschaftlicher Perspektive. Göttingen (= Wissenschaft und Lehrerbildung 2), 151-174.

Graf, Margot / Vogel-Waldhütte, Martina / Halbartschlager, Franz ( $\left.{ }^{4} 2018\right)$ : MEHRfach Geschichte 4. Teil 1-Wissen \& Verstehen. Linz.

HAMMERSTEIN, KATRIN (2017): Gemeinsame Vergangenheit - getrennte Erinnerung? Der Nationalsozialismus in Gedächtnisdiskursen und Identitätskonstruktionen von Bundesrepublik Deutschland, DDR und Österreich. Göttingen (= Diktaturen und ihre Überwindung im 20. und 21. Jahrhundert 11).

HoBSBAwm, ERIC (1995): Das Zeitalter der Extreme. Weltgeschichte des 20. Jahrhunderts. München / Wien.

JACOBMEYER, WolfGANG (1998): Das Schulgeschichtsbuch - Gedächtnis der Gesellschaft oder Autobiographie der Nation? In: Geschichte, Politik und ihre Didaktik 26:26-35.

Kershaw, IAN (2016): Höllensturz. Europa 1914 bis 1949. Bonn (= Schriftenreihe 1780).

KLIMPFINGER, ViKTORIA (2018): „Anschluss “. Ein Wort auf Gänsefüßchen. In: Wiener Zeitung v. 01.03.2018: https://www.wienerzeitung.at/nachrichten/kultur/mehr_kultur /949969_Ein-Wort-auf-Gaensefuesschen.html?em_cnt_page=1 (06.08.2019).

KüHBerger, Christoph / Neureiter, Herbert (2017): Zum Umgang mit Nationalsozialismus, Holocaust und Erinnerungskultur. Eine quantitative Untersuchung bei Lernenden und Lehrenden an Salzburger Schulen aus geschichtsdidaktischer Perspektive. Schwalbach/Ts. (= Geschichte unterrichten).

Mader, Sabine / Strutz, Andrea (2019): querdenken 4. Geschichte und Politische Bildung. Wien.

MARKOVA, INA (2015a): „Geschichtsbilder“ in österreichischen Lehrmaterialien 1978/79. Sagbarkeits- und Zeigbarkeitsregeln am Beispiel „Anschluss“. In: Bildungsforschung 12, 1:34-53. 
MARKOVA, INA (2015b): Österreichische Schlüsselbilder der NS-Zeit. Visuelle Gedächtnisse und deren Wandel in Geschichtsschulbüchern der Zweiten Republik. In: KÜHBERger, CHRISTOPH / MitTNIK, PHILIPP: Empirische Geschichtsschulbuchforschung in Österreich. Innsbruck / Wien / Bozen (= Österreichische Beiträge zur Geschichtsdidaktik 10), 119-134.

Melichar, Franz / PlatTner, IRMGard / RAUCHEGGER-Fischer, Claudia / ScHARINGER, StEPHAN (2018): GO! Geschichte Oberstufe 7/8. Neuer Lehrplan. Wien.

MitTNIK, PHILIPP / KÜHBERGER, CHRISTOPH (2015): Geschichtsschulbuchforschung in Österreich - Splitter der rezenten Entwicklung. In: KÜHBERGER, CHRISTOPH / MITTNIK, PHILIPP (eds.): Empirische Geschichtsschulbuchforschung in Österreich. Innsbruck / Wien / Bozen (= Österreichische Beiträge zur Geschichtsdidaktik 10), 9-18.

MitTNIK, PHILIPP (2017): Holocaust-Darstellung in Schulbüchern. Deutsche, österreichische und englische Schulbücher im Vergleich. Schwalbach/Ts.

Monyk, Elisabeth / Schreiner, Eva / MAnn, Elisabeth (2010): Geschichte für alle. 4. Klasse. Wien.

Monyk, Elisabeth / Schreiner, Eva (2019): Geschichte für alle 4 Modular. Wien.

OleschKo, Sven / Moraitis, Anastasia (2012): Die Sprache im Schulbuch. Erste Überlegungen zur Entwicklung von Geschichts- und Politikschulbüchern unter Berücksichtigung sprachlicher Besonderheiten. In: Bildungsforschung 9, 1:11-46.

Paireder, Bettina / Hofer, Jutta (2019): Meine Geschichte 4. Module für Geschichte, Sozialkunde und Politische Bildung. Linz.

PANDEL, HANS-JÜRGEN (2005): Was macht ein Schulbuch zu einem Geschichtsbuch. Ein Versuch über Kohärenz und Intertextualität. In: HANDRO, SASKIA / SCHÖNEMANN, BERND (eds.): Geschichtsdidaktische Schulbuchforschung. Berlin (= Zeitgeschichte - Zeitverständnis 16), 15-37.

PANDEl, HANS-JÜRgEN (2017): Geschichtsdidaktik. Eine Theorie für die Praxis. Schwalbach/Ts.

RAthKolb, Oliver (2011): Die paradoxe Republik. Österreich 1945 bis 2010. Innsbruck.

RothFELS, HANS (1956): Zeitgeschichte als Aufgabe. In: Vierteljahreshefte für Zeitgeschichte 1:1-8.

RÜSEN, JÖRN (1983): Historische Vernunft. Grundzüge einer Historik I: Die Grundlagen der Geschichtswissenschaft. Göttingen.

RÜSEN, JÖRN (1994): Historisches Lernen. Grundlagen und Paradigmen. Köln / Weimar / Wien.

RÜSEN, JÖRN (2008): Historisches Lernen. Grundlagen und Paradigmen.

SCHÖNEMANN, BERND / THÜNEMANN, HOLGER (2010): Schulbucharbeit. Das Geschichtslehrbuch in der Unterrichtspraxis. Schwalbach/Ts.

Schuschnigg, KuRT ( $\left.{ }^{2} 1978\right)$ : Ein Requiem in Rot-Weiß-Rot. Mit einem Vorwort von Stephan Verosta. Wien. 
Staudinger, Eduard / Ebenhoch, Ulrike / Scheucher, Alois / Scheipl, Josef (2019a): Zeitbilder 7. Vom Ende des Ersten Weltkrieges bis in die Gegenwart. Wien.

Staudinger, Eduard / Ebenhoch, Ulrike / Scheucher, Alois / Scheipl, Josef (2019b): Zeitbilder 7/8. Vom Ende des Ersten Weltkrieges bis in die Gegenwart. Wien.

Staudinger, Eduard / Ebenhoch, Ulrike / Scheucher, Alois / Scheipl, Josef (2014): Zeitbilder 7. Vom Ende des Ersten Weltkrieges bis in die Gegenwart. Wien.

Uhl, HeIDEMARIE (2004): Die Transformation des ,"österreichischen Gedächtnisses “ in der Erinnerungskultur der Zweiten Republik. In: Geschichte und Region / Storia e regione 13, 2:23-54.

Wolfrum, Edgar (1999): Geschichtspolitik in der Bundesrepublik. Der Weg zur bundesrepublikanischen Erinnerung 1948-1990. Darmstadt. 